Voix et Images

voixetimages

\title{
Oeuvres de Pierre Perrault
}

\section{François Cormier et Yves Lacroix}

Volume 3, numéro 3, avril 1978

\section{Pierre Perrault}

URI : https://id.erudit.org/iderudit/200117ar

DOI : https://doi.org/10.7202/200117ar

Aller au sommaire du numéro

\section{Éditeur(s)}

Les Presses de l'Université du Québec

ISSN

0318-9201 (imprimé)

1705-933X (numérique)

Découvrir la revue

Citer ce document

Cormier, F. \& Lacroix, Y. (1978). Oeuvres de Pierre Perrault. Voix et Images, 3(3), 371-378. https://doi.org/10.7202/200117ar d'utilisation que vous pouvez consulter en ligne.

https://apropos.erudit.org/fr/usagers/politique-dutilisation/ 


\section{OEuvres de Pierre Perrault}

\section{Articles au Quartier-Latin}

Nadja II, 22 octobre 1948, p. 2.

Sur l'intellectualisme, 18 février 1949, p. 1.

Opinions sur l'homme, 22 février 1949, p: 1.

La Justice n'est pas à demander mais à rendre, $1^{\text {er }}$ mars 1949, p. 1.

Visages cramoisis, 4 mars 1949, p. 1.

Comptabilité, 8 mars 1949, p. 3.

Bilan de l'an carabin, 22 mars 1949, p. 1.

"Tit-Coq" à l'université, 22 mars 1949, p. 6.

Bienvenue aux Carabins, 4 octobre 1949, p. 1.

Opinions, 7 octobre 1949, p. 1.

Avant d'écrire un éditorial, 11 octobre 1949, p. 1.

On dépense à s'étriquer, 14 octobre 1949, p. 1.

Réponse s'il vous plaît, 21 octobre 1949, p. 1.

L'Avenir de l'esprit, 2 novembre 1949, p. 1.

Bergeronnales, 4 novembre 1949, p. 2.

Samedi dernier, 4 novembre 1949, p. 4.

Les Petits Lundis de L'A.G.E.U.M., 22 novembre 1949, p. 1.

Glissades, 25 novembre 1949, p. 3.

Un gros lundi, 2 décembre 1949, p. 1-2.

Gála fantaisiste, 9 décembre 1949, p. 1-2.

Un lundi comique, 13 décembre 1949, p. 1-2.

Prière de Noël, 16 décembre 1949, p. 4-5.

Trois Lettres de l'alphabet, 3 février 1950, p. 1.

Le Sens de l'humour, 10 février 1950, p. 1.

"Les Assassins sont parmi nous ", 10 février 1950, p. 3.

La Famine menace les rats de bibliothèque, 14 février 1950, p. 1. 
L'Étudiant chrétien en face du monde actuel, 17 février 1950, p. 1-2. Nous, la politique et les politiciens, 21 février 1950, p. 1.

Carrefour 50, 24 février 1950, p. 1-2.

Lundi musical, 28 février 1950, p. 1.

L'Université libre, avec Marc Brière, 3 mars 1950, p. 1-2.

La Faculté des lettres, les idées et les mots, 3 mars 1950, p. 1.

Récit d'un voyageur, 7 mars 1950, p. 1-4.

Nous la religion et..., 21 mars 1950, p. 1.

Parade, 22 septembre 1950, p. 4.

Deux Séminars en Europe, avec Yolande Simard, 19 décembre 1950, p. 2.

\section{2. Émissions radiophoniques}

Au bord de la rivière (d'octobre 1955 à février 1956). Émission hebdomadaire de Radio-Canada. Réalisation de Jacques Bertrand; lecture de Raymond Charette; interprétation d'Hélène Baillargeon, Monique Chailler et Jacques Labrecque ; orchestre de Hector Gratton.

Le Chant des hommes (d'avril 1956 à mars 1958). Émission quotidienne de Radio-Canada. Réalisation de Madeleine Martel; lecture de François Bertrand.

Poèmes et chansons (octobre et novembre 1956). Émission hebdomadaire de Radio-Canada. Réalisation de Jacques Douai.

Au pays de Neufve-France (de novembre 1956 à octobre 1957). Émission hebdomadaire de Radio-Canada. Réalisation de Madeleine Martel; lecture de François Bertrand; interprétation de Jacques Douai.

Noëls anciens. Un texte créé à Radio-Canada le 25 décembre 1957 avec des chansons de Jacques Douai. Réalisation de Madeleine Martel. L'émission sera reprise le 25 décembre 1958.

Destination inconnue (de mars à septembre 1958). Émission quotidienne (sept jours par semaine) de Radio-Canada. Réalisation de Madeleine Martel; lecture de François Bertrand.

La Violette double doublera (de janvier à décembre 1959). Émission hebdomadaire de Radio-Canada. Réalisation de Madeleine Martel; lecture de François Bertrand.

Chroniques de terre et de mer, première série (de janvier à juin 1960). Émission hebdomadaire de Radio-Canada. Réalisation de Madeleine Martel; lecture de François Bertrand.

Ballades du temps précieux (de janvier à juillet 1961). Émission hebdomadaire de Radio-Canada. Réalisation de Lorenzo Godin; interprétation de Stephen Fentok, Monique Chailler, Monique Miville-Deschênes; lecture de Raymond Charette.

Imagerie sur ma ville (de juillet à septembre 1961). Émission hebdomadaire de Radio-Canada. Réalisation de Madeleine Martel; lecture de Raymond Charette. 
Paysages de la chanson (de août à décembre 1969). Émission hebdomadaire de Radio-Canada. Réalisation de Jean-Yves Contant; interprétation du Quatuor des Jeunesses musicales et du Petit Ensemble vocal.

Noël à l'île-aux-Coudres. Un texte d'une demi-heure créé à RadioCanada le 25 décembre 1961.

L'Hiver à l'île-aux-Coudres. Un texte d'une heure créé à Radio-Canada le 25 avril 1962.

Chroniques de terre et de mer, deuxième série (d'octobre 1963 à juin 1964). Émission hebdomadaire de Radio-Canada. Réalisation de Bernard Vanasse; lecture de Pierre Perrault.

J'habite une ville (de janvier à septembre 1965). Émission hebdomadaire de Radio-Canada. Réalisation de Bernard Vanasse; lecture de Pierre Perrault. Elle sera reprise d'octobre 1965 à juin 1966.

Gens de mon pays (de juin à septembre 1971). Série de onze émissions animées par Diane Giguère et Pierre Perrault. Réalisation de Pierre Rainville.

\section{Dramatiques pour la télévision}

La Lettre, la Loge et le Repas frugal, sketches inspirés par Vermeer, Renoir et Picasso pour la série Musée intime, donnés à Radio-Canada le 8 juillet 1956. Réalisation de Paul Blouin; animation de Guy Viau et Françoise Faucher; interprétation de Denise Provost et Jean Brousseau. L'émission sera reprise dans la série Télé-archives de Radio-Canada le 19 juillet 1971.

L'Anse aux huards, créé à Radio-Canada le 20 juillet 1958 dans la série Théâtre d'été. Réalisation de Paul Blouin; interprétation de Yves Létourneau, Jean-Pierre Masson, Hélène Loiselle et Albert Millaire.

Au cœur de la rose, créé à Radio-Canada le 30 novembre 1958, dans là série En Première. Réalisation de Paul Blouin; interprétation de Monique Miller, François Guillier, Edmond Beauchamp, Marthe Thiéry, Marcel Cabay et Albert Millaire. Arrangements musicaux de _Stephen Fentok. L'émission sera reprise le 22 mars 1959.

Vent d'ès, créé à Radio-Canada le 12 juin 1960 dans la série En première. Réalisation de Paul Blouin; interprétation de Monique Miller, Guy Hoffman, Andrée Lachapelle, André Cailloux, Madeleine Sicotte, Lucille Cousineau et Colette Courtois.

Adaptation de la Maison de Bernarda Alba de Federico Garcia Lorca, créée à Radio-Canada le 14 mars 1963. Réalisation de Paul Blouin.

\section{Dramatiques pour la scène}

$J$ 'habite une ville, poème dramatique. Créé à l'école des Beaux-Arts en 1962 par le Centre d'essai. Mise en scène de Natan Karczmar. 
Les Frères Colin, poème dramatique. Créé à l'école des Beaux-Arts. en 1963 par le Centre d'essai. Mise en scène de Natan Karczmar.

Au cœur de la rose, pièce en trois actes. Créée par les Apprentis-Sorciers le 7 février 1963. Mise en scène de. Jean-Guy Sabourin. Interprétation de Micheline Pelland, André Richard, Serge Turgeon, Yolande Marchessault, Jean-Guy Sabourin, Pierre Cuillerier et François Beaulieu.

C'est. l'enterrement de Nicodème tout le monde est invité, pièce en trois actes. Créée par les Apprentis-Sorciers au Troisième Festival international du théâtre amateur, tenu à Monaco du 4 au 13 septembre 1965. La première canadienne eut lieu le 9 octobre 1965 au Centre culturel Saint-Jean-Eudes à Québec, dans le cadre du Congrès national de l'ACTA. Mise en scène de Jean-Guy Sabourin. Interprétation de Jeanne Leroux, Pierre Cuillerier, Jean-Pierre Saulnier, Pierre Collin, Yolande Marchessault et Monique Duplantie-Paradis.

\section{Livres}

Portulan, poèmes, Montréal, Beauchemin, 1961.

Toutes is/es, chroniques, Montréal, Fidès, 1963. Nouvelle édition : Montréal, Fidès, "Bibliothèque canadienne-française», 1966.

Ballades du temps précieux, poèmes, illustration de Anne Trèze. Montréal, éd. d'Essais, 1963. Nouvelle édition en 1965.

$A u$ cœur de la rose, pièce en trois actes, première version, illustration de Claude Sabourin, Montréal, éd. des Apprentis-Sorciers, 1963, numéroté de 1 à 256.

Au coeur de la rose, pièce en trois actes, deuxième version, illustration de Claude Sabourin, Montréal, éd. Beauchemin, 1964. Cette édition sera reprise par Lidec Inc., à Móntréal en 1969.

Lè Règne du jour, transcription du film, Montréal, Lidec Inc., 1968.

Les Voitures d'eau, transcription du film, Montréal, Ici-Radio-Canada/Lidec Inc., 1969.

En désespoir de cause, poèmes de circonstances atténuantes, Montréal, Parti pris, «Paroles», $n^{\circ} 18,1971$.

Un pays sans bon sens, transcription du film, commentaires et notes de Pierre Perrault, Montréal, Lidec Inc., 1972.

Chouennes, poèmes de 1961 à 1971. Comprend Portulan, Ballades du temps précieux, En désespoir de cause et quelques inédits, y compris la Chansons de Marie déjà parue dans le Règne du jour, Montréal, I'Hexagone, 1975.

Gélivures, poème, Montréal, l'Hexagone, 1977.

Discours sur la condition sauvage et québécoise, photos et témoignages rassemblés par Pierre Perrault, Montréal, Lidec Inc., 1977. 


\section{Autres publications}

"Les Pierres en vrac", pièce en deux tableaux, dans les Cahiers d'Arlequin, Montréal, collège Sainte-Marie, 1947.

"Suite et fin", dans le Devoir, 29 octobre 1949, p. 9.

"La faculté de droit ne s'intéresse pas au droit", avec Marc Brière, dans l'Autorité, $38^{\mathrm{e}}$ année, $n^{\circ} 46$, Beauceville, 6 juin 1953, p. 1.

"Le Jazz", dans les Cahiers radiophoniques, Société Radio-Canada, vol. 1, $n^{\circ} 1$ (avril 1958), p. 39-42.

"René Richard», dans les Cahiers radiophoniques, Société Radio-Canada, vol. $1, n^{\circ} 1$ (avril 1958), p. 73-79.

"Noël ancien dans une vallëe nouvelle», dans le Devoir, 22 décembre 1962 , p. 9.

"Préface" à un recueil sans titre des étudiants du collège Notre-Dame, 1962-1963.

“J'habite une ville», dans Liberté, $n^{\circ} 28$ (juillet-août 1963), p. 375-384.

"Chanson des voyageurs du temps passé en vain", dans Lettres et écritures, vol. 1, $\mathrm{n}^{\circ} 1$ (décembre 1963), p. 21-30.

"La Mi-Carême à l'île-aux-Coudres", dans Maclean, mars 1964, p. 25 et suiv. Photos de Michel Brault.

"Préface" à un recueil sans titre des étudiants du collège Notre-Dame, 1963-1964.

"Lettre aux demoiselles du collège Gourgeois ", dans Lettres et écritures, vol. $2, n^{\circ} 1$ (novembre 1964), p. 10-11.

"L'écrivain", dans Lettres et écritures, vol. 2, n 1 (novembre 1964), p. 19.

"J'habite une ville", dans le Devoir, 19 juin 1965, p. 31.

Fragment autographe du manuscrit de la première version de $A u$ cceur de la rose, reproduit en fac-similé dans le programme du spectacle des Apprentis-Sorciers, Au cœur de la rose, Pierre Perrault, Montréal, 1963, p. 16. "Filet crevé", chanson écrite en 1956 avec Jacques Douai pour la série Au pays de Neufve-France, dans Rimes et chansons de Roger Girod, Stockholm, Sveriges Radio, 1965, p. 93-94.

“Discours sur la parole», dans Culture vivante, $\mathrm{n}^{\circ} 1$ (1966), p. 19-36. Photos de Michel Brault. Aussi dans les Cahiers du cinéma, $n^{\circ} 191$ (juin 1967), p. 26-33. Aussi dans Ar Vro, $n^{\circ} 44$ (1 ${ }^{\mathrm{er}}$ novembre 1967), p. 80-97.

“Lettre de Montréal ", dans les Cahiers du cinéma, n 194, octobre 1967. p. 59-62.

"Québec", épilogue à Option-Québec de René Lévesque, Montréal, éd. de l'Homme, 1968, p. 170-173.

"Cinéma et réalité", dans Comment faire ou ne pas faire un film québécois, Montréal, La Cinémathèque canadienne, 1968.

“Gélivures", extrait, dans Culture vivante, n 12 (février 1969), p. 42-43. 
Réponse à l'enquête de Cécile Cloutier-Wojciechowska, «Témoignages des poètes canadiens-français", Archives des lettres canadiennes, tome IV, Montréal, Fidès, 1959, p. 557-560.

"Gélivures", extrait, dans Archives des lettres canadiennes, tome IV, Montréal, Fidès, 1959 , p. 661.

"La Violette double doublera", préface au Chant de l'alouette de Raoul Roy, Québec, Les Presses de I'Université Laval/lci-Radio-Canada, 1969. p. 4.

«Poèmes du temps présent », dans Maintenant, Montréal, $\mathrm{n}^{\circ} 101$ (décembre 1970), p. 306-307.

«Se donner des outils de réflexion, dans Cinéma Québec, vol. $1, n^{\circ} 1$ (mai 1971), p. 20-21.

Extrait d'une lettre inédite, datée du 28 octobre 1965, cité dans Une approche de la poésie québécoise de notre temps de Marcel Colin, Saint-Jean, Richelieu, 1971, p. 24.

«Pierre Perrault parle de Un pays sans bon sens», dans la revue du cinéma Image et son, $n^{\circ} 256$ (janvier 1972), p. 43-44.

"Prendre la parole pour briser le silence", dans l'Art et l'État, en collaboration avec Robert Roussil et Denis Chevalier, Montréal, Parti pris, 1973, p. 65-101.

“Préface » au Mushuau nipi, à l'âge du cäribou de Louis-Edmond Hamelin, Québec, Centre d'études nordiques, Université Laval, 1973, p. iii-xii.

“A propos des voitures d'eau ", postface à Marins du Saint-Laurent de Gérard Harvey, Montréal, éd. du Jour, 1974, p. 245-310.

"A propos de cette pièce", dans le Théâtre populaire du Québec, vol. 1, $n^{\circ} 1$, automne 1974, p. 2.

"L'Apprentissage de la haine", dans l'Homme dans son nouvel environnement, IV Colloque, Session Ross 1974, Université du Québec à Rimouski, décembre 1974, p. 17-48.

«Préface" à l'Acadie du discours de Jean-Paul Hautecœur, Québec, Les Presses de l'Université Laval, 1975, p. ix-xxv.

«Entretien "; dans Cinéastes du Québec, $n^{\circ} 5$, Montréal, Conseil québécois pour la diffusion du cinéma, septembre 1970, p. 16-48.

Réponse à l'enquête «Témoignages sur le théâtre québécois», Archives des lettres canadiennes, tome V, Montréal, Fidès, 1976, p. 780.

«Défiance», photo d'un poème manuscrit, le vingt et unième feuillet d'une série de textes publiés à Montréal en 1976 au profit des Grands Ballets canadiens, sous le titre 100000 Prétextes pour danser.

"Lettre du Québec ", dans Pourquoi?, Paris, Ligue française de l'enseignement et de l'éducation permanente, $n^{\circ} 121$ (janvier 1977), p. 39-49.

«Discours sur la condition sauvage et québécoise", dans Terre Québec théâtre de la grande réplique, Montréal, vol. 1, $n^{\circ} 2$ (janvier-février 1977), p. 6 . 
"Trou d'homme", dans Bernard Gosselin, rétrospective, avril-mai 1977, Montréal, la Cinémathèque québécoise, p. 5-8. Le texte est repris dans le Jour, 29 juillet 1977, p. 29-31.

"De l'artisanat comme instrument de conquête", dans Possibles, Montréal, vol. 1, n³/4 (printemps-été 1977), p. 59-97.

"Les Hommes chantent la mort", dans Essais et erreurs, revue non datée, p. 26-31.

"Le Royaume des pères à l'encontre des fils, le.Devoir, 28 janvier 1978, p. 33 et 48 .

\section{Films}

Maîtres-artisans du Canada. Commentaire de Pierre Perrault. Production de Crawley Films, 1957. Coul.

La Légende du Corbeau. Commentaire de Pierre Perrault. Production de Crawley Films, 1957. Coul.

Au pays de Neufve-France: Réalisation et montage de René Bonnière: images de Michel Thomas d'Hoste, Alan Grayston, Kenneth Campbell, Stanley Brede et Frank Stokes; musique de Larry Crosly; chansons de Monique Miville-Deschênes; narration de François Bertrand; commentaire de Pierre Perrault; direction de la production par Pierre Perrault, production de Crawley Films, 1959-1960.

Série de treize films de trente minutes:

Le Traverse d'hiver à l'île-aux-coudres, n. \& b.; Attiuk, le Jean-Richard, Tête-a-la-baleine, l'Anse Tabatière, Ka-ke-ki-ku, Anse-aux-Basques, En revenant de Saint-Hilarion, Diamants du Canada, les Goélettes, Rivière du Gouffre, la Pitoune et Toutes isles, coul.

Pour la suite du monde. Réalisation de Pierre Perrault et Michel Brault; images de Michel Brault et Bernard Gosselin; son de Marcel Carrière; musique de Jean Cousineau, production de l'O.N.F., 105 min., n. \& b., 1963. Le Règne du jour. Réalisation de Pierre Perrault; images de Bernard Gosselin et Jean-Claude Labrecque; son de Serge Beauchemin et Alain Dostie, musique de Jean-Marie Cloutier, production de l'O.N.F., $118 \mathrm{~min}$., n. \& b., 1966.

Les Voitures d'eau. Réalisation de Pierre Perrault; images de Bernard Gosselin; son de Serge Beauchemin; montage de Monique Fortier ; production de l'O.N.F., 110 min., n. \& b., 1969.

Le Beau Plaisir. Réalisation de Pierre Perrault; images de Bernard Gosselin et Michel Brault; son de Claude Pelletier, Serge Beauchemin et Sydney Pearson; musique de Aimé Gagnon, Raymond Gagnon et Jean-Baptiste Gagnon; production de l'O.N.F., $15 \mathrm{~min}$., coul., 1969.

Un pays sans bon sens. Réalisation de Pierre Perrault; images de Bernard Gosselin; son de Serge Beauchemin; montage de Yves Leduc; production de l'O.N.F., 117 min., n. \& b., 1970. 
L'Acadie l'Acadie. Réalisation de Pierre Perrault et Michel Brault; images de Michel Brault; son de Serge Beauchemin; montage de Monique Fortier, production de l'O.N.F., n. \& b., 1970.

Un royaume vous attend. Réalisation de Pierre Perrault; images de Bernard Gosselin; son de Claude Beaugrand et Claude Chevalier; montage de Suzanne Dussault; production de l'O.N.F., 110 min., 1975

Le Goot de la farine. Réalisation de Pierre Perrault; images de Bernard Gosselin; son de Serge Beauchemin, Jacques Chevigny et Claude Beaugrand; montage de Monique Fortier; production de l'O.N.F., $117 \mathrm{~min}$., coul., 1977.

Le Retour à la terre. Réalisation de Pierre Perrault; images de Bernard Gosselin; son de Claude Beaugrand et Claude Chevalier; montage de Claire Boyer; production de l'O.N.F., 56 min. coul., 1977.

C'était un Québécois en Bretagne, madame. Réalisation de Pierre Perrault; images de Bernard Gosselin; son de Claude Beaugrand; montage de Claire Boyer; production de l'O.N.F., 60 min., coul., 1977.

(Document préparé par François Cormier et Yves Lacroix.) 